\title{
Observation of weakly and strongly diverging ion beams in a magnetically expanding plasma
}

\author{
K. Takahashi ${ }^{\mathrm{a})}$ and T. Fujiwara \\ Department of Electrical and Electronic Engineering, Iwate University, Morioka 020-8551, Japan
}

(Received 2 December 2008; accepted 20 January 2009; published online 9 February 2009)

\begin{abstract}
The spatial distribution of an ion beam created in a magnetically expanding plasma using permanent magnets is experimentally investigated for 0.35 and $1 \mathrm{mTorr}$, where the magnetic-field strength is about $100 \mathrm{G}$ in the plasma source and is decreasing into a few gauss in the diffusion chamber. The beam profile for 0.35 mTorr is weakly divergent. On the other hand, the strongly diverging beam is detected for $1 \mathrm{mTorr}$. The results are discussed from the viewpoint of the plasma-potential structures and imply the beam divergence caused by the radial electric fields in the ion acceleration region and the diffusion chamber. (C) 2009 American Institute of Physics. [DOI: 10.1063/1.3080205]
\end{abstract}

Plasma-potential structures in plasmas have been studied for a long time in connection with the acceleration and deceleration of the charged particles in space and laboratory plasmas. ${ }^{1-5}$ Since the formation of an electric double layer (DL) and the subsequent ion acceleration in expanding helicon plasmas were recently reported, ${ }^{6,7}$ much attention is focused on the magnetically and geometrically expanding plasmas $^{8-13}$ in the electric propulsion community because it would lead to the new type of electric thruster without any electrode. Typical subjects of interest to this community are the power consumption and the spatial distribution of the accelerated ion beam. In order to reduce power consumption in the magnetically expanding source, authors have already reported a solenoid-free plasma magnetically expanded by only permanent magnets (PMs), ${ }^{14}$ where a DL potential drop can be created near the source exit and a supersonic ion beam can be detected in the diffusion chamber. Regarding the spatial distribution of the ion beam, spatially resolved energy analyzer measurements have been reported. The results in the magnetically expanding plasma have shown the weakly diverging ion beam ${ }^{15,16}$ while the strongly diverging one has been detected in the geometrically expanding plasma, ${ }^{17}$ where it is important to know what decides the beam divergence.

In the present letter, we report the spatial investigation of the ion beam generated by the DL in the magnetically expanding plasma using PMs, where a weakly diverging ion beam is changed into a strongly diverging one when the operating gas pressure is increased from 0.35 to $1 \mathrm{mTorr}$. The variation in the spatial distribution of the ion beam is discussed from the viewpoint of the existence of the radial electric field.

A schematic of the experimental setup is shown in Fig. 1(a), which has previously described. ${ }^{14}$ Briefly, an expanding plasma source, consisting of a $20-\mathrm{cm}-$ long and $6.5-\mathrm{cm}-$ diameter cylindrical glass tube surrounded by a double-turn loop antenna situated at $z=-9 \mathrm{~cm}$ and powered from an $\mathrm{rf}$ generator of $13.56 \mathrm{MHz}$ and $250 \mathrm{~W}$, is connected contiguously to a $26-\mathrm{cm}$-diameter and $30-\mathrm{cm}$-long grounded vacuum chamber (diffusion chamber), where $z=0$ is defined as the outlet of the source tube. The double concentric arrays of neodymium iron boron $(\mathrm{NdFeB})$ magnets surrounding the

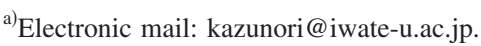

source tube provide an expanding magnetic field of $\sim 100 \mathrm{G}$ in the source $(z \sim-13$ to $-5 \mathrm{~cm})$ and a few gauss in the middle of the diffusion chamber $(z=15 \mathrm{~cm})$. Argon gas is introduced from the source side via a mass flow controller and the pressure in the chamber is maintained at 0.35 or 1 mTorr.

The plasma-potential structures and the ion beam characteristics are investigated by three movable retarding field energy analyzers (RFEAs), which consist of an electron reflector mesh biased at $-90 \mathrm{~V}$, an ion collector electrode, and a 3-mm-diameter entrance orifice, as shown in Fig. 1(a), where the local plasma potential and the ion energy distribution function (IEDF) can be obtained by the RFEAs with orifice facing radially and with orifice facing the source side, respectively. Two RFEAs (RFEA 1 and RFEA 3) with orifice facing radially can provide the axial profile of the plasma potential on axis, and the radial profiles at various axial positions in the range of $z \geq 3 \mathrm{~cm}$, respectively. The axially and radially movable RFEA facing the source tube (RFEA 2) gives us the spatial distribution of the IEDF or the ion beam. We should mention that the local plasma potential measured

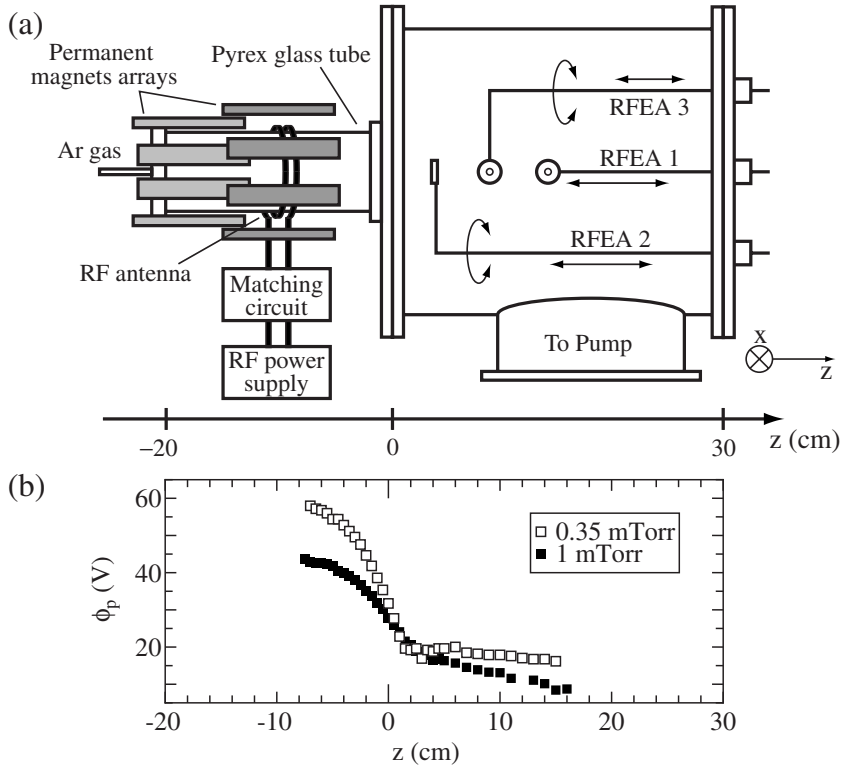

FIG. 1. (a) Schematic diagram of experimental setup. (b) Axial profiles of the plasma potential $\phi_{p}$ for 0.35 mTorr (open squares) and $1 \mathrm{mTorr}$ (closed squares). 
(a)

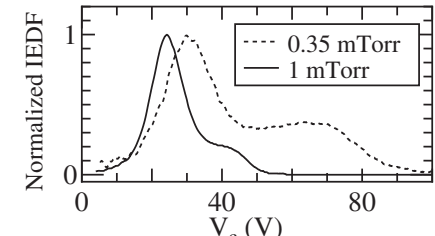

(b)

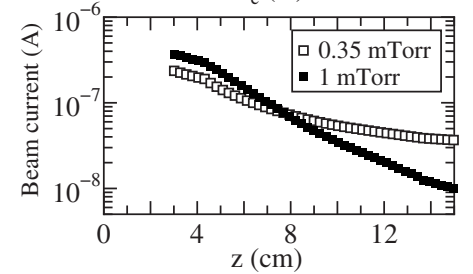

FIG. 2. (a) Normalized IEDFs for 0.35 mTorr (dashed line) and 1 mTorr (solid line). (b) Axial profile of the beam current flowing into the collector electrode for 0.35 mTorr (open squares) and 1 mTorr (closed squares), respectively.

by the RFEA facing radially is shifted to the low-energy side compared with one facing the source tube as reported in another experiments. ${ }^{13}$ However, we can know the relative plasma potential structure by the RFEA facing radially and the beam energy by the RFEA facing the source tube.

Figure 1(b) shows the axial profiles of the plasma potential $\phi_{p}$ measured by RFEA 1 on axis for 0.35 mTorr (open squares) and 1 mTorr (closed squares). As previously described, ${ }^{14}$ the rapid potential drop of the DL with thickness of 100-200 Debye length is spontaneously formed near the outlet of the source tube $(z=-2-1 \mathrm{~cm})$ for 0.35 mTorr. This rapid potential drop is also observed for 1 mTorr, as shown in Fig. 1(b), although the potential difference between the source tube and the diffusion chamber becomes small. This result resembles the behavior of the current-free DL reported previously. ${ }^{10,18}$ Here, it is noted that the local plasma potential for $1 \mathrm{mTorr}$ gradually decreases in the downstream side of the DL $(z \geq 1 \mathrm{~cm})$. This potential gradient originates from the density gradient, i.e., Boltzmann's law. In other words, it is considered that the potential structures following Boltzmann's law is superimposed on the DL structure for higher gas pressure condition of 1 mTorr.

With the axially facing RFEA 2 located at $z=3 \mathrm{~cm}$ downstream of the DL, the IEDFs for 0.35 and $1 \mathrm{mTorr}$ are obtained, as shown in Fig. 2(a). In both cases, the IEDFs show the accelerated group of ions (right peak) at collector bias voltage $V_{c} \sim 65$ and $40 \mathrm{~V}$ for 0.35 and $1 \mathrm{mTorr}$ in addition to the bulk ions (left peak) at $V_{c} \sim 30$ and $23 \mathrm{~V}$ for 0.35 and $1 \mathrm{mTorr}$. The energies of the accelerated beam ions for 0.35 and 1 mTorr are about 35 and $17 \mathrm{eV}$, respectively, and are in good agreement with the potential drop of the DL, where the beam ions for $1 \mathrm{mTorr}$ are additionally accelerated by Boltzmann electric field downstream of the DL $(z$ $\geq 1 \mathrm{~cm}$ ). Measurements of spatial profiles of the beam current flowing into the collector electrode of the RFEA, which is proportional to the beam density, are performed, where the collector bias voltage $V_{c}$ is maintained at 55 and $37 \mathrm{~V}$ for 0.35 and $1 \mathrm{mTorr}$, respectively. These collector bias voltages are selected for detecting only the beam ions. Following the principle of the RFEA measurement, the measured collector current profiles indicate the spatial distribution of the accelerated ions coming from the high-potential upstream plasma. Figure 2(b) shows the axial profiles of the beam current flowing into the collector electrode for 0.35 mTorr (open squares) and 1 mTorr (closed squares), where the beam cur-
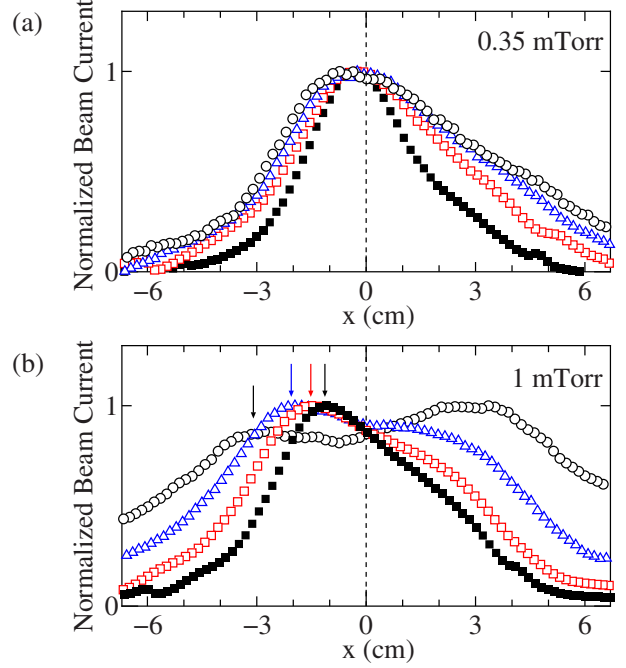

FIG. 3. (Color online) $x$ profiles of the beam current flowing into the collector electrode at $z=3 \mathrm{~cm}$ (closed squares), $5 \mathrm{~cm}$ (open squares), $7 \mathrm{~cm}$ (open triangles), and $9 \mathrm{~cm}$ (open circles) for (a) $0.35 \mathrm{mTorr}$ and (b) $1 \mathrm{mTorr}$.

rents decay exponentially with a decay constant of about 7 and $2.5 \mathrm{~cm}$, which are inversely proportional to the gas pressure and close to the ion-neutral mean free path for charge exchange as reported previously. ${ }^{19}$

In order to know the spatial distributions of the accelerated ions, the radial profiles of the beam current for 0.35 and $1 \mathrm{mTorr}$ are measured at various axial positions. Figures 3(a) and 3(b) show the normalized $x$ profiles of the beam current obtained at $z=3 \mathrm{~cm}$ (closed square), $5 \mathrm{~cm}$ (open squares), $7 \mathrm{~cm}$ (open triangles), and $9 \mathrm{~cm}$ (open circles), for 0.35 and 1 mTorr, respectively, where $x=0$ is defined as the radial center. It is found in Fig. 3(a) that the divergence of the ion beam is very small for $0.35 \mathrm{mTorr}$, although it is difficult to estimate the divergence angle. On the other hand, the result for 1 mTorr shown in Fig. 3(b) does clearly show the strongly diverging ion beam, where the peak and the edge of the beam current is shifted to the radially outward side in the downstream side. Here, the asymmetries of the beam profiles in Fig. 3 would originate from the nonuniformity of the upstream plasma. The improvement of the rf antenna is required. The position of the peaks observed in the range of $x<0$ are indicated as arrows in Fig. 3(b), which is discussed later.

For discussing the mechanisms of the above-mentioned gas-pressure-induced change from the weakly diverging beam to the strongly diverging one, the measurements of the radial profiles of the plasma potential are performed in the diffusion chamber. Figure 4 shows $x$ profiles of the plasma potential $\phi_{p}$ and the radial electric field $E_{r}$ at (a) $z=3 \mathrm{~cm}$, (b) $z=5 \mathrm{~cm}$, and (c) $z=8 \mathrm{~cm}$ for $0.35 \mathrm{mTorr}$ (open squares) and $1 \mathrm{mTorr}$ (closed squares). For $0.35 \mathrm{mTorr}$, the potential profiles are almost flat and there is no radial electric field in the range of $|x|<3 \mathrm{~cm}$, which corresponds to the radius of the source tube. The measured results for 1 mTorr clearly show the radial gradient of the plasma potential and the radial electric field of about $0.5-2 \mathrm{~V} / \mathrm{cm}$ at the center area, which would give rise to divergence of the ion beam by modifying the orbit of the beam ions. Furthermore, the radial electric field near the source exit $(z=3 \mathrm{~cm})$ appears to be larger than the downstream side $(z=8 \mathrm{~cm})$. It is expected that the radial electric field exists even inside the DL potential drop at 

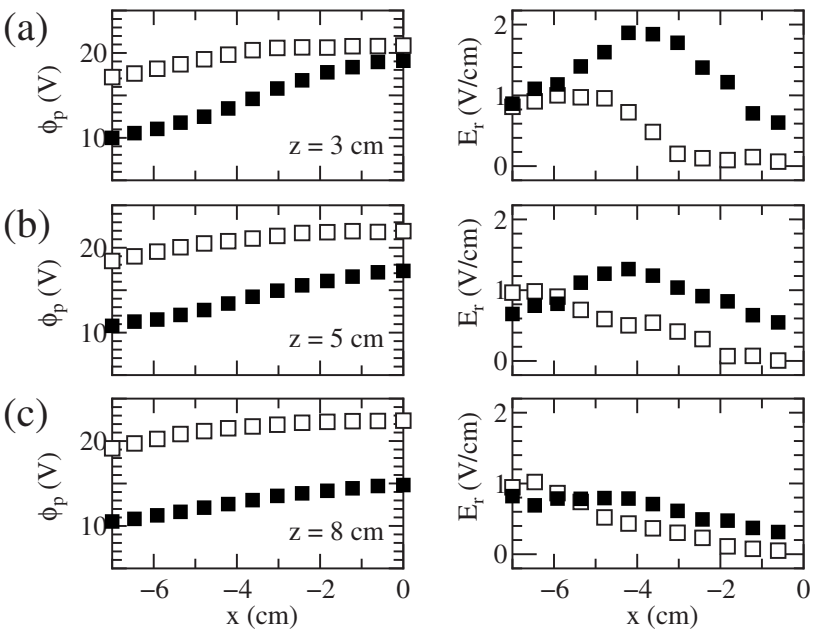

FIG. 4. $x$ profiles of the plasma potential $\phi_{p}$ and the radial electric field $E_{r}$ measured at (a) $z=3 \mathrm{~cm}$, (b) $5 \mathrm{~cm}$, and (c) $8 \mathrm{~cm}$ for 0.35 mTorr (open squares) and 1 mTorr (closed squares), respectively.

$z=-2-1 \mathrm{~cm}$ and inside the source tube for $1 \mathrm{mTorr}$, which implies the transition from a plane DL structure without radial electric field to a convex DL structure when the operating gas pressure is increased. As previously suggested, ${ }^{17}$ the convex potential structure can also cause the diverging ion beam. The measurement of the two-dimensional potential structure including the DL and the source tube is our next challenge.

In the last paragraph, we suggested the two generation mechanisms of the strongly diverging ion beam for 1 mTorr. One is the modification of the orbit of the beam ions, which have already accelerated through the DL, by the radial electric field downstream of the DL. Another is the generation of the diverging beam by the convex DL structure. These are discussed using a simple kinetic equation of the single ion particle, which has already been accelerated by the DL and has a axial velocity of $v_{\|}=\sqrt{2 e \phi_{\mathrm{DL}} / M_{i}}$. The kinetic equation in the radial direction derives the radial position $r_{i}$ of the ion particle for the case that the ion drifts a distance $z_{D}$ in the axial direction during a time $t$, as $r_{i}=\left(e / 2 M_{i}\right) E_{r} t^{2}+v_{r 0} t$, where $M_{i}, E_{r}$, and $v_{r 0}$ are the ion mass, the radial electric field in the diffusion chamber, and the initial radial velocity of the ion. When $z_{D}$ is the distance measured from the end of the DL, we have $t=z_{D} / v_{\|}$. Substituting for $t$ in the above formula, we can obtain

$$
r_{i}=\frac{e}{2 M_{i}} E_{r}\left(\frac{z_{D}}{v_{\|}}\right)^{2}+\frac{v_{r 0}}{v_{\|}} z_{D}
$$

The first term shows the radial drift by the radial electric field in the diffusion chamber, which is observed for $1 \mathrm{mTorr}$ in Fig. 4. The second term originates from the radial acceleration in the DL structure, i.e., beam divergence effect by the convex DL. In order to roughly estimate the ion orbit, the radial positions $r_{\text {peak }}$ of the peaks indicated as arrows in Fig. 3 (b) are plotted in Fig. 5 as open squares, where $z_{D}$ is estimated as $z_{D}=z-1 \mathrm{~cm}$ because the end of the observed DL is $z \sim 1 \mathrm{~cm}$. The dashed line shows the first term in Eq. (1) for $E_{r}=1 \mathrm{~V} / \mathrm{cm}$, which is roughly estimated by Fig. 4. It is found that this first term cannot explain the beam divergence, but the radial field plays an important role in the beam divergence. In order to fit Eq. (1) to the experimental data, the second term in Eq. (1) for $v_{r 0} / v_{\|}=0.3$ needs to be included in

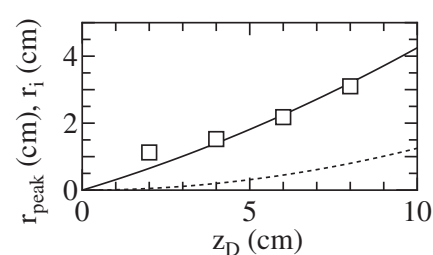

FIG. 5. Radial positions $r_{\text {peak }}$ (open squares) of the peaks in the beam current profiles for 1 mTorr [arrows in Fig. 3(b)] depending on the axial distance $z_{D}$ from the end of the DL, together with the estimated radial position $r_{i}$ of the ion particle from the first term (dashed line) and the sum of the first and second terms (solid line) in Eq. (1), respectively.

Eq. (1), where the calculated result is plotted as solid line in Fig. 5. Thus, we suggest that the beam divergence for higher gas pressure of $1 \mathrm{mTorr}$ is caused by both the convex DL structure and the radial electric field downstream of the DL. Here, we need to mention that the factor $v_{r 0} / v_{\|}=0.3$ indicates the radial acceleration of about $1.5 \mathrm{eV}$ during the axial acceleration of $17 \mathrm{eV}$ in the DL because of $v_{r 0} / v_{\|}$ $=\sqrt{\varepsilon_{r} / \phi_{\mathrm{DL}}}$, where $\varepsilon_{r}$ is the radial energy of the accelerated ions. Hence, the generation of weakly diverging beam requires to produce the plasma with the radially flat potential profile.

In summary, we observed the weakly and strongly diverging ion beams in the magnetically expanding plasma using PMs for very low pressure of 0.35 mTorr and higher pressure of 1 mTorr, respectively. It is described that the beam divergence would be caused by both the convex DL structure and the radial electric field downstream of the DL for higher gas pressure even in the magnetically expanding plasma. The results indicate that the low-pressure operation of this source is suitable for an efficient propulsion device.

This work is partially supported by Grant-in-Aid for Young Scientists (Grant No. B, 20740317) from the Ministry of Education, Culture, Sports, Science and Technology, Japan, and by TEPCO Research Foundation.

${ }^{1}$ G. Hairapetian and R. L. Stenzel, Phys. Rev. Lett. 61, 1607 (1988).

${ }^{2}$ C. Charles, Plasma Sources Sci. Technol. 16, R1 (2007).

${ }^{3}$ K. Takahashi, T. Kaneko, and R. Hatakeyama, Appl. Phys. Lett. 91, 261502 (2007).

${ }^{4}$ A. Fruchtman, Phys. Rev. Lett. 96, 065002 (2006).

${ }^{5}$ D. S. Main, D. L. Newman, and R. E. Ergun, Phys. Rev. Lett. 97, 185001 (2006).

${ }^{6}$ C. Charles and R. Boswell, Appl. Phys. Lett. 82, 1356 (2003).

${ }^{7}$ S. A. Cohen, N. S. Siefert, S. Stange, R. F. Boivin, E. E. Scime, and F. M. Levinton, Phys. Plasmas 10, 2593 (2003).

${ }^{8}$ X. Sun, A. M. Keesee, C. Biloiu, E. E. Scime, A. Meige, C. Charles, and R. W. Boswell, Phys. Rev. Lett. 95, 025004 (2005).

${ }^{9}$ F. F. Chen, Phys. Plasmas 13, 034502 (2006).

${ }^{10}$ M. A. Lieberman and C. Charles, Phys. Rev. Lett. 97, 045003 (2006).

${ }^{11}$ C. Charles and R. W. Boswell, Appl. Phys. Lett. 91, 201505 (2007).

${ }^{12}$ I. A. Biloiu, E. E. Scime, and C. Biloiu, Appl. Phys. Lett. 92, 191502 (2008).

${ }^{13}$ C. S. Corr, J. Zanger, R. W. Boswell, and C. Charles, Appl. Phys. Lett. 91, 241501 (2007).

${ }^{14}$ K. Takahashi, K. Oguni, H. Yamada, and T. Fujiwara, Phys. Plasmas 15, 084501 (2008).

${ }^{15}$ C. Charles, IEEE Trans. Plasma Sci. 33, 336 (2005).

${ }^{16}$ W. Cox, C. Charles, R. W. Boswell, and R. Hawkins, Appl. Phys. Lett. 93, 071505 (2008).

${ }^{17}$ C. S. Corr, R. W. Boswell, C. Charles, and J. Zanger, Appl. Phys. Lett. 92, 221508 (2008).

${ }^{18}$ O. Sutherland, C. Charles, N. Plihon, and R. W. Boswell, Phys. Rev. Lett. 95, 205002 (2005).

${ }^{19}$ M. D. West, C. Charles, R. W. Boswell, and J. Propul, Power 24, 134 (2008). 